\title{
Behavioral specifications of reward-associated long-term memory enhancement in humans
}

\author{
Bianca C. Wittmann, ${ }^{1,5,6}$ Raymond J. Dolan, ${ }^{1}$ and Emrah Düzel ${ }^{2,3,4}$ \\ ${ }^{1}$ Wellcome Trust Centre for Neuroimaging, University College London, London WC1N 3BC, United Kingdom; ${ }^{2}$ Institute of Cognitive \\ Neuroscience, University College London, London WC1N 3AR, United Kingdom; Institute of Cognitive Neurology and Dementia \\ Research, Otto-von-Guericke-Universität Magdeburg, Magdeburg 39120, Germany; ${ }^{4}$ German Centre for Neurodegenerative \\ Diseases-Magdeburg, Otto-von-Guericke-Universität Magdeburg, Magdeburg 39120, Germany
}

\begin{abstract}
Recent functional imaging studies link reward-related activation of the midbrain substantia nigra-ventral tegmental area (SN/VTA), the site of origin of ascending dopaminergic projections, with improved long-term episodic memory. Here, we investigated in two behavioral experiments how (1) the contingency between item properties and reward, (2) the magnitude of reward, (3) the uncertainty of outcomes, and (4) the contextual availability of reward affect long-term memory. We show that episodic memory is enhanced only when rewards are specifically predicted by the semantic identity of the stimuli and changes nonlinearly with increasing reward magnitude. These effects are specific to reward and do not occur in relation to outcome uncertainty alone. These behavioral specifications are relevant for the functional interpretation of how reward-related activation of the SN/VTA, and more generally dopaminergic neuromodulation, contribute to long-term memory.
\end{abstract}

Reward improves long-term memory formation in incidental and intentional encoding paradigms (Wittmann et al. 2005; Adcock et al. 2006; Callan and Schweighofer 2008; Wittmann et al. 2008). This reward-related memory enhancement is associated with co-activation of substantia nigra-ventral tegmental area (SN/VTA), striatum, and hippocampus as seen in functional magnetic resonance imaging (fMRI) studies (Wittmann et al. 2005; Adcock et al. 2006; Wittmann et al. 2008). This pattern of activation implicates dopaminergic neuromodulation as a mediating influence on this enhancement. It is well-established that dopamine increases and prolongs hippocampal long-term potentiation (LTP) in animals (Frey et al. 1990, 1991; Huang and Kandel 1995; Bernabeu et al. 1997; Sajikumar and Frey 2004; Lemon and Manahan-Vaughan 2006), while antagonists of dopamine D1/D5 receptors impair hippocampus-dependent memory consolidation ( $\mathrm{O}^{\prime} \mathrm{C}$ arroll et al. 2006). Given that reward-predicting stimuli elicit a dopaminergic response (see Schultz 2007), rewardrelated manipulation of long-term memory can serve as a model for investigating the dopaminergic neuromodulatory regulation of plasticity in long-term memory.

Despite increasing evidence for dopaminergic modulation of hippocampal memory, little is known about the specific behavioral variables which drive and control reward-related memory enhancement. Specifically, existing behavioral paradigms leave open how (1) item-reward relationship, (2) reward magnitude, (3) outcome uncertainty, and (4) contextual effects of reward availability contribute to memory enhancement. Item-reward relationship is determined by which feature of a studied item predicts reward be it core features such as the meaning (e.g., semantic category) of items or peripheral features (e.g., color). We hypothesized that coupling the category of study items with reward

${ }^{5}$ Present address: Institute of Psychology, University of Giessen, Otto-Behaghel-Str. 10, 35394 Giessen, Germany. ${ }^{6}$ Corresponding author.

E-mail bianca.wittmann@psychol.uni-giessen.de; fax 496419926159. Article is online at http://www.learnmem.org/cgi/doi/10.1101//m.1996811. availability would enhance memory relative to that seen with coupling of reward and peripheral item features. This hypothesis was based on task efficiency considerations. For value learning in the real world, an agent has to evaluate the contingencies between different features of an event and the event's consequences (the "outcome"). The most successful and most efficient strategy is to identify and preferentially process only those features that are connected to the motivational outcome. In the color group, the words were arbitrarily assigned to color/motivational status, and semantic processing of the words did therefore not contribute to performing the rewarded task. Enhancing memory for these irrelevant semantic features would not be advantageous. In the category group, in contrast, extracting the meaning of the words was directly relevant to preparation for the target task. Better memory for the words strengthens category distinctions in this group and would be likely to enhance performance on a repeat version of the task.

Reward magnitude could in principle influence encoding in two ways: the fidelity of memory enhancement could be high enough to linearly reflect even moderate increases in reward magnitude. Alternatively, the enhancement could have low fidelity leading to nonlinearities through insensitivity for moderate increases of reward magnitude. A related issue is outcome uncertainty, a possible confound in reward studies. To keep participants engaged and maintain high cue-related dopaminergic activity, the probability of gaining in reward trials is usually not $100 \%$. This potentially creates an expectation of perceptual uncertainty in reward trials (gain/loss feedback), which could affect memory formation through an impact on attention or arousal (Chun and Turk-Browne 2007).

The contextual effect of reward availability refers to the possibility that, in a learning context containing reward, there may be two types of memory enhancement: a strong specific enhancement for reward-predicting stimuli and a diffuse, unspecific enhancement for other stimuli in the same context. There are two mechanisms that could lead to a diffuse and contextual effect. One is related to tonic changes in dopamine availability 
Behavioral reward effects on memory

that integrate the availability of reward over many trials (Niv et al. 2007). Another is related to the phenomenon of synaptic capture (Frey and Morris 1997; Morris 2006; Frey and Frey 2008). Dopamine induces the synthesis of plasticity-related proteins, which remain available intracellularly for several minutes. These proteins can be "captured" by other synapses to stabilize memory for other stimuli following the dopamine-releasing event (Frey and Morris 1997; Morris 2006; Frey and Frey 2008).

To test the contribution of these factors to reward-related memory enhancement, we performed two group studies based on an established rewarded memory paradigm (Fig. 1; Wittmann et al. 2005, 2008; Krebs et al. 2009). Experiment 1 assessed the effects of reward magnitude and item-reward relationship. Twenty-four participants were randomly assigned to two groups of 12 participants (six men, six women each). In group 1 (the "color group") the reward status of each trial was indicated by a peripheral stimulus feature, namely the color of the cue word (red, blue, or green). In group 2 (the "category group"), the reward status of each trial was indicated by stimulus identity, namely the semantic category of the cue word ("humans," "animals and plants," or "objects"). In both groups, there were three levels of reward magnitude (no reward, $€ 0.20, € 0.40$ ). Experiment 2 addressed the contribution of uncertainty and the contextual effect of reward availability. Thirty-one participants were randomly assigned to two groups of 15 (seven men) and 16 (six men) participants. Participants in group 1 (the "reward group") performed a standard rewarded memory paradigm with two reward levels (no reward or $€ 0.50$ ). Participants in group 2 (the "uncertainty group") performed the same task with cues predicting two types of feedback: uncertain (two possible outcome symbols) or certain (one outcome symbol).

\section{Results}

Only trials with correct responses were entered into the analysis of reaction times because of group differences in the overall number of correct and incorrect responses. In the study phase in experiment 1, reward-predicting cues led to a speeding of participants' reaction times (RTs) for the number task in correct rewarded trials (mean low reward \pm SE: $471 \pm 14$ msec; mean high reward: $471 \pm 15 \mathrm{msec}$ ) compared to correct unrewarded trials (mean no reward \pm SE: $490 \pm 13 \mathrm{msec}$ ) irrespective of whether the prediction was by identity or color (two-way ANOVA with three reward levels and two experimental groups, main effect of reward: $F_{(2,44)}=12.22 ; P<0.001$; no interaction). For the word category decision, there was no difference in RT between rewarded and nonrewarded trials in either group, no interaction, and no overall group difference (Table 1 ; main effect of reward: $F_{(2,44)}=2.22, P=0.12$; interaction: $F_{(2,44)}=0.61, P=0.55$; group difference: $\left.F_{(1,22)}=1.14 ; P=0.3\right)$.

We tested the effect of reward on recollection- and familiarity-based memory performance (Table 2; Fig. 2) in a three-way

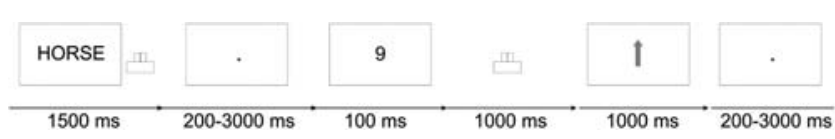

Figure 1. Experimental design. Trial sequence for the study phase, shown exemplarily for a rewarded trial in experiment 2. A cue word was presented indicating whether participants could win money on that trial. Participants made a category decision on the word, waited for the following number task, and then indicated quickly whether the number was higher or lower than five. In rewarded trials, they received feedback after correct decisions made within a time limit. In neutral trials, they did not receive meaningful feedback.
Table 1. Category classification reaction times in experiment 1

\begin{tabular}{lccc}
\hline & $\begin{array}{c}\text { No } \\
\text { reward }\end{array}$ & $\begin{array}{c}\text { Medium } \\
\text { reward }\end{array}$ & $\begin{array}{c}\text { High } \\
\text { reward }\end{array}$ \\
\hline Color group RT (msec) & $858 \pm 41$ & $850 \pm 42$ & $837 \pm 41$ \\
Category group RT (msec) & $938 \pm 40$ & $897 \pm 36$ & $877 \pm 44$ \\
\hline
\end{tabular}

ANOVA with the within-subjects parameters memory condition ("remember" /"know") and reward (no reward/20 cents/40 cents) and the between-subjects parameter of group ("color" / "category"). There was no main effect of reward on memory $\left(F_{(2,44)}=0.27, P=\right.$ $0.74)$. We found an interaction effect of memory condition $x$ reward $\left(F_{(2,44)}=3.4, P<0.05\right)$. This was mainly driven by the category group, reflected in a three-way interaction effect (memory condition $\times$ reward $\times$ group $\left.\left[F_{(2,44)}=6.6, P<0.05\right]\right)$. We also found an interaction of memory condition $\times$ group $\left(F_{(1,22)}=4.5, P<0.05\right)$ caused by a higher overall remember rate in the category group than in the color group (post-hoc $t$-test $P<0.001)$. There was a trend-level overall memory difference between groups (main effect of group: $F_{(1,22)}=3.5, P=0.08$ ) that was mostly driven by the rewarded items. A post-hoc $t$-test on only the unrewarded items did not reveal significant differences for the remember or know rates (remember rate $t_{(22)}=1.34$, $P=0.19$; know rate $\left.t_{(22)}=1.1, P=0.28\right)$, although the difference in overall hit rate between the category and color groups was nearly significant $\left(t_{(22)}=2.07, P=0.051\right)$.

In separate ANOVAs on each group the category group showed a significant increase in remember rate for rewarded items (memory condition $\times$ reward $F_{(2,22)}=3.9, P<0.05$ ), in contrast to the color group, which did not show this effect $\left(F_{(2,22)}=2.2\right.$, $P=0.15)$. There was no significant effect of reward on false alarm rates in the category group $\left(F_{(2,22)}=0.95, P=0.40\right)$. It was not possible to test for this effect in the color group because distractors were shared between reward categories. False alarm rates did not differ between groups $\left(t_{(22)}=0.38, P=0.71\right)$.

In the study phase in experiment 2 , RTs (RT data of one participant were lost) for the number comparison task were significantly shorter in correct rewarded trials (mean \pm SE: $340 \pm 13$ msec) than in correct unrewarded trials (378 $\pm 13 \mathrm{msec}$; paired $t$-test, $t_{(13)}=-5.78, P<0.001$ two-tailed). In the uncertainty group, RTs (note that RT data of 12 participants were lost) were significantly shorter in correct uncertain trials $(868 \pm 31 \mathrm{msec})$ than in correct certain trials (962 $\pm 30 \mathrm{msec}$; paired $t$-test, $t_{(3)}=-6.77$, $P<0.01$ two-tailed). It should be noted as a caveat that the RT comparison in the uncertainty group included data from only four subjects (data from the remaining 12 subjects could not be used because of technical difficulties). Word category decisions were also faster in rewarded $(688 \pm 24 \mathrm{msec})$ than nonrewarded trials (764 $\pm 24 \mathrm{msec}$; paired $t$-test, $t_{(13)}=-6.53, P<0.001$ twotailed) and faster in uncertain $(866 \pm 31 \mathrm{msec})$ vs. certain trials (963 \pm 30 msec; paired $t$-test, $t_{(3)}=-8.13, P<0.01$ two-tailed).

For the memory test in experiment 2 (Table 3; Fig. 3), a repeated-measures ANOVA on corrected hit rates revealed a trendlevel interaction of the combined "reward and uncertainty" factor with group $\left(F_{(1,29)}=2.98, P=0.095\right)$. We further found this interaction in an ANOVA on remember rates $\left(F_{(1,29)}=4.12, P=0.055\right)$. Post-hoc paired $t$-tests revealed a higher remember rate for rewarded than unrewarded items in the reward group $\left(t_{(14)}=\right.$ $2.22, P<0.05$ two-tailed), but no effect of uncertainty on remember rate in the uncertainty group $\left(t_{(15)}=-0.6, P=0.58\right)$. There was no difference between the unrewarded items in the reward group and "certain" or "uncertain" items in the uncertainty group on any memory measure ( $t$-tests, all $P>0.2)$. False alarm rates did not differ between the groups $\left(t_{(29)}=0.50, P=0.62\right)$. 
Table 2. Memory performance in experiment 1

\begin{tabular}{llccc}
\hline & & $\begin{array}{c}\text { No } \\
\text { reward }\end{array}$ & $\begin{array}{c}\text { Medium } \\
\text { reward }\end{array}$ & $\begin{array}{c}\text { High } \\
\text { reward }\end{array}$ \\
\hline Corr. remember & Color group & $11.4 \pm 2$ & $11.4 \pm 2$ & $13.6 \pm 2$ \\
$\quad$ rate (\%) & Category group & $16.0 \pm 2$ & $24.2 \pm 4$ & $24.7 \pm 3$ \\
Corr. know rate (\%) & Color group & $14.6 \pm 3$ & $16.8 \pm 3$ & $11.9 \pm 2$ \\
& Category group & $19.8 \pm 4$ & $10.2 \pm 3$ & $15.5 \pm 5$ \\
Corr. hit rate (\%) & Color group & $26.0 \pm 4$ & $28.2 \pm 3$ & $25.5 \pm 3$ \\
& Category group & $35.8 \pm 3$ & $34.3 \pm 4$ & $40.2 \pm 5$ \\
\hline
\end{tabular}

\section{Discussion}

These experiments provide evidence for semantic identity specific enhancement of recollection by reward association. Recollection is considered a hippocampus-dependent form of long-term memory (Vargha-Khadem et al. 1997; Duzel et al. 2001; Yonelinas et al. 2005) and our findings therefore provide new data regarding the specific circumstances in which reward-related manipulations enhance hippocampus-dependent plasticity. When reward cues were linked to peripheral stimulus features and semantic identity was nonpredictive of rewards, reward did not modulate memory formation. This lack of reward modulation was evident despite the fact that participants were processing the meaning of each stimulus and that reward anticipation shortened reaction times (in the number task). This suggests that memory enhancement is strongest if semantic identity and reward status are directly associated and is compatible with the hypothesis that it is computationally more efficient to enhance memory specifically for features that are task-relevant.

Another possible interpretation of these findings could be that a dissociation of reward status and encoding tasks leads to dual-task demands: participants pay attention to the peripheral reward cues while also performing the deep encoding task. Divided attention is known to impair memory performance (Chun and Turk-Browne 2007). This would explain the generally lower memory performance in the color group. However, we found no evidence for divided attention in an RT comparison of the tasks (Table 1) and therefore do not think that divided attention effects were strong enough to explain the absence of a reward effect nor the trend-level overall lower memory performance in the color group. The trend toward overall better performance in the category group may point toward the interesting possibility that, when elaborative processing is incentivized by future rewards, encoding is improved. Testing such a possibility requires further experiments.

In this study, reward magnitude had a nonlinear effect: Doubling the amount of reward predicted by a stimulus from 20 cents to 40 cents did not increase recollection. However, since we could not include a larger range of values in order to keep the memory demands practicable, it is not possible to infer that the relationship between reward and memory performance is generally nonlinear. A possible interpretation of our finding is that dopaminergic neuromodulation may not have sufficient fidelity to differentially respond to 20 and 40 cents. Although studies in monkeys show that dopaminergic neurons can adapt their gain and fidelity to capture small differences in reward magnitude (Tobler et al. 2005), the experimental circumstances leading to such adaptivity were different from those used here. When these specific conditions are replicated in humans, small differences in reward magnitude can cause strong differences in memory performance. Specifically, in a recent study reward-predicting cues could be followed by two possible reward outcomes (Bunzeck et al. 2010). Pictures were presented after the reward outcome and did not serve as cues in contrast to the current experiment. This experiment closely followed one conducted in nonhuman primates which showed that activity in dopaminergic circuitry did not signal the absolute prediction error but rather an adaptively scaled, binary version of it in terms of "better" or "worse" of two possible outcomes (Tobler et al. 2005). Under such conditions, memory modulation by rewarding outcomes followed an adaptively scaled, binary pattern of prediction in mesolimbic circuitry (Bunzeck et al. 2010). Adaptive coding of rewards has not yet been shown for reward-predicting stimuli. In the Bunzeck et al. (2010) study, differences in expected value of the reward cues were not represented by activity differences in the dopaminergic system at cue time. At outcome time, participants were required to indicate whether the outcome was the better or worse of two options (Bunzeck et al. 2010). It is possible that this decision process sharpens the neural representation of reward values in contrast to processing at cue time in the current study.

Both incidental and intentional reward memory tasks typically involve an element of outcome uncertainty: Not all items that indicate reward availability are later rewarded. Uncertainty can itself be a salient signal with alerting and attention-enhancing properties (Vickery and Jiang 2009; but see Yu and Dayan 2005; Grinband et al. 2006). In experiment 2, uncertainty led to shorter RTs on both tasks, indicating increased attention or arousal. Despite this, uncertainty expectation had no effect on longterm memory, supporting the idea that memory enhancement by reward anticipation is driven by reward and, by inference, by reward-related dopamine release. Our results therefore support the hypothesis entertained in previous studies (Wittmann et al. 2005; Adcock et al. 2006) that reward-related memory enhancement is related to the motivational aspects of reward and cannot be explained by outcome uncertainty per se. However, studies in animals show that when outcome uncertainty is very high (50\% reward probability) there is a ramping up of dopaminergic firing in the time window between the cue signalling the uncertain outcome and the outcome itself (Fiorillo et al. 2003). Our data do not rule out the possibility that under similar conditions of high uncertainty there could be a specific memory modulation by uncertainty itself. Because animal studies always include a reward component, it is still unclear whether uncertainty in the absence of reward causes a similar dopaminergic response. Although our results do not conclusively address this possibility, experiment 2 did provide a control for the uncertainty levels typically present in reward trials and supports the conclusion that the memory effect in these trials is driven by reward and not by uncertainty.

According to the framework of "synaptic capture" or "behavioral tagging" (Frey and Morris 1997; Morris 2006; Frey and Frey 2008; Ballarini et al. 2009), events that cause dopamine release should modulate memory for other items presented in the same context. For rewards, this possibility could not be assessed in

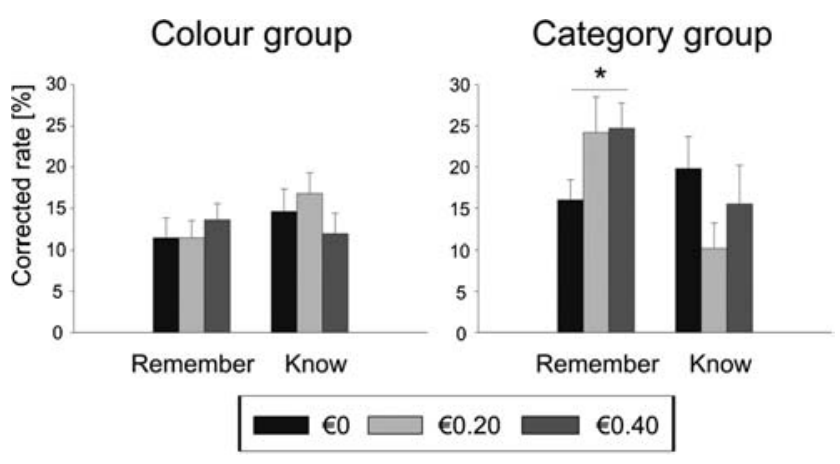

Figure 2. Memory performance in experiment 1. 
Table 3. Memory performance in experiment 2

\begin{tabular}{llcc}
\hline & & $\begin{array}{c}\text { No reward/ } \\
\text { certainty }\end{array}$ & $\begin{array}{c}\text { Reward/ } \\
\text { uncertainty }\end{array}$ \\
\hline Corr. remember & Reward group & $12.8 \pm 4$ & $21.8 \pm 2$ \\
rate (\%) & Uncertainty group & $18.8 \pm 3$ & $16.4 \pm 2$ \\
Corr. know rate (\%) & Reward group & $19.1 \pm 3$ & $19.2 \pm 3$ \\
& Uncertainty group & $14.0 \pm 3$ & $14.2 \pm 2$ \\
Corr. hit rate (\%) & Reward group & $31.9 \pm 5$ & $41.0 \pm 4$ \\
& Uncertainty group & $32.8 \pm 4$ & $30.5 \pm 2$ \\
\hline
\end{tabular}

previous experiments that compared reward-predicting items with neutral items from the same (rewarding) context. These experiments only provided evidence for a specific reward effect on individual items but could not rule out the possibility that memory for the "neutral" items was enhanced compared to a context in which no reward was available at all. In our direct betweensubjects contrast, memory for neutral ("certainty-predicting") items from the rewarding context vs. the comparable item class ("certainty-predicting items") from the uncertainty context showed no difference on any memory measure, confirming that reward exerts a specific effect on those items meaningfully connected to its delivery. However, we cannot fully rule out the possibility that a within-subjects design might reveal context effects that were potentially obscured by the between-subjects design of experiment 2 .

This result seemingly conflicts with other studies that used novelty and suggest that brief dopaminergic activity can lead to an enhancement of subsequent hippocampal plasticity over a period of several minutes ( $\mathrm{Li}$ et al. 2003; Fenker et al. 2008; Ballarini et al. 2009). These studies report lasting effects of novelty on subsequent hippocampal plasticity (Li et al. 2003; Ballarini et al. 2009) and hippocampus-dependent memory for stimuli occurring within 30 min after exposure to novelty (Fenker et al. 2005; Ballarini et al. 2009). Our findings suggest that such contextual effects of memory enhancement are more prominent with novelty than with reward, but more studies are needed to confirm this. However, from the vantage point of the physiology of dopamine firing, a difference between novelty and reward is not entirely unexpected. Hippocampal novelty signals are likely to increase the pool of tonically active dopamine neurons via a hippocampal-VTA circuit (Goto and Grace 2008). In contrast, rewards are more likely to cause phasic bursts of dopaminergic activity (Schultz 2002; Lisman and Grace 2005; Duzel et al. 2010). Exposure to novelty could thus lead to a period of elevated tonic firing which may not occur to the same extent with exposure to rewards. Because only dopamine neurons that are in tonic firing mode can be excited into burst-firing (Goto and Grace 2008), the consequence of such a difference between novelty and reward could be that stimuli occurring a few minutes after novelty exposure are more likely to elicit a burst-firing response than those occurring after exposure to rewards. A second factor to consider is that Fenker et al. (2008) tested novelty effects on memory for familiar ("weak") items, while our reward task and the control uncertainty task contained only novel items. We cannot exclude the possibility that contextual memory enhancement may occur for rewards if all the stimuli used are pre-familiarized.

These experiments show that the mechanism through which reward improves episodic memory formation is highly stimulus and feature specific. From an evolutionary point of view, such specificity provides a plausible mechanism for allowing accurate source memory for reward-predicting events. In humans, memory enhancement through motivational factors such as rewards has potential therapeutic relevance for memory impairment in aging and in neurological and psychiatric conditions where it is speculated there is limbic and dopaminergic pathology.
Understanding the specific behavioral factors that govern such enhancement will contribute substantially toward developing effective interventions.

\section{Materials and Methods}

The experiments were conducted in accordance with guidelines of the ethics committee of the University of Magdeburg, Faculty of Medicine. All words were taken from the CELEX database (Baayen et al. 1995) and matched for word length and frequency. All participants received written instructions.

The structure of both experiments was identical. During each trial (Fig. 1), participants saw a trial-unique German word for 1500 msec, responded to it with a button press (right index or middle finger), waited a variable interval (delay, 200-3000 msec duration), and then responded to a number (target, $100 \mathrm{msec}$ ) by a button press. Visual feedback (1000 msec duration) was given 1000 msec after presentation of the target. The next trial followed after a variable fixation phase (200-3000 msec). The speeded number comparison task (Wittmann et al. 2005) required participants to decide whether the target number $(1,4,6$, or 9) was lower or higher than 5 . They responded as quickly as possible by button press with their right index or middle finger. A response time limit was used to determine trial outcome.

In reward trials, participants received negative feedback (no money) if their response to the target number was incorrect or after the response time limit. After correct decisions within the time limit, they received positive feedback. The time limit was adjusted individually in a staircase procedure to ensure a reward rate of $\sim 75 \%$. Participants were aware of the speed-accuracy requirements and reward contingencies. Frequency of target buttons and numbers was counterbalanced for each session. Participants were asked to pay attention to the cues to ensure awareness of the reward/uncertainty status of each trial, but not told that a memory test would follow.

In experiment 1 , the study session consisted of 150 trials (Fig. 1). Fifty trials were potentially rewarded with $€ 0.20,50$ trials with $€ 0.40$, and 50 trials were neutral (no reward available). Participants in the category group indicated for each cue whether they expected a low reward, a high reward, or no reward (based on the categories "human", "animals and plants", and "objects"). Participants in the color group made a semantic decision on the word (living or nonliving) to avoid a confound by levels-ofprocessing effects, where word color predicted the three reward outcomes.

In experiment 2 , the study session consisted of 120 trials. In the reward group, 60 trials were potentially rewarded with $€ 0.50$, and 60 trials were neutral. The reward status of each trial was indicated by word category ("humans" or "animals and plants"). In the uncertainty group, participants did not receive experimental rewards (but were compensated for their time with a fixed payment). The study session was divided into 60 trials that contained outcome uncertainty and 60 trials with certain outcomes. The uncertainty status of each trial was indicated by word category ("humans" or "animals and plants"). Outcomes consisted of three
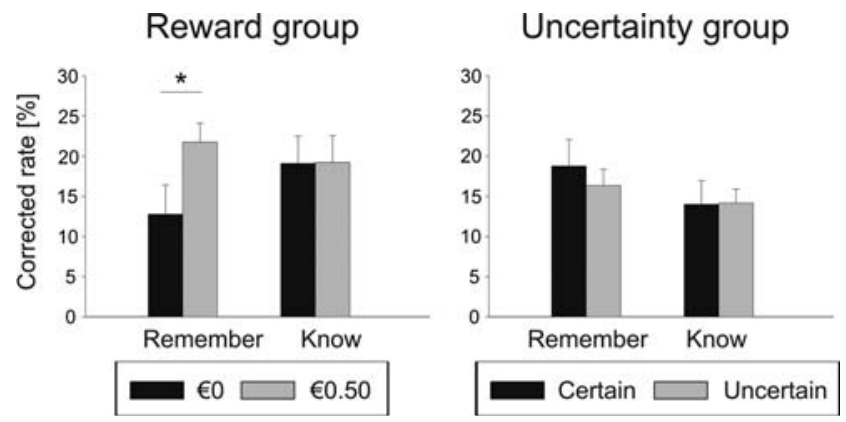

Figure 3. Memory performance in experiment 2. 
symbols: a green square or a red circle in uncertain trials, and a black triangle in certain trials. Participants were instructed to indicate for each word whether they expected a "green/red" or "black" outcome. Presentation of the outcomes was not contingent on participants' responses to the number task. In order to match the task features to those of the reward group, the green square was presented in $75 \%$ and the red circle in $25 \%$ of uncertain trials. Outcomes in certain trials always consisted of the black triangle. To ensure participants' attention to the outcomes, they were asked to estimate the percentage of green outcomes in the middle and at the end of the study session.

In all groups, a memory test was conducted on the next day. Participants were shown all words from the study phase randomly mixed with newly presented distractor words (75 in experiment 1, 60 in experiment 2, corresponding to half the number of old words). Participants received written instructions and the task was self-paced. First, participants indicated whether they recognized the word ("Old/New"). If they did, they then judged their memory according to the remember/know procedure ("Remember/Know/Guess") (Tulving 1985; Duzel et al. 1997). For words classified as new, participants indicated whether their decision was confident ("Sure/Guess"). In experiment 1, participants in the color group also indicated whether an "old" word had been associated with no, low, or high reward in the study phase. As the reward category was indicated by semantic category in group 2 and in both groups in experiment 2 , a source memory judgment was not possible on the basis of reward category in these groups. Participants may of course have used other aspects of the study episode to recollect source information about old items. Response time limits were set at $3 \mathrm{sec}$ for the first and $2.5 \mathrm{sec}$ for the second (and third) decision. A fixation phase of $1 \mathrm{sec}$ duration followed. Every 60-76 trials, the task was paused until participants were ready to continue.

By adding the remember and know rates for old stimuli (percentage of studied items classified as remembered or known), and subtracting the corresponding false alarm rate for distractors (percentage of unstudied items classified as remembered or known), we obtained corrected hit rates. We also calculated a corrected remember rate and a corrected know rate separately by subtracting the corresponding false alarm rates. Note that these response rates excluded trials in which participants guessed.

\section{Acknowledgments}

We thank Isabell Zlobinski and Katrin Arndt for assistance with data acquisition.

\section{References}

Adcock RA, Thangavel A, Whitfield-Gabrieli S, Knutson B, Gabrieli JD 2006. Reward-motivated learning: Mesolimbic activation precedes memory formation. Neuron 50: 507-517.

Baayen RH, Piepenbrock R, Gulikers L. 1995. The CELEX lexical database. Release 2 [CD-ROM]. Linguistic Data Consortium, University of Pennsylvania, Philadelphia.

Ballarini F, Moncada D, Martinez MC, Alen N, Viola H. 2009. Behavioral tagging is a general mechanism of long-term memory formation. Proc Natl Acad Sci 106: 14599-14604.

Bernabeu R, Bevilaqua L, Ardenghi P, Bromberg E, Schmitz P, Bianchin M, Izquierdo I, Medina JH. 1997. Involvement of hippocampal cAMP/ cAMP-dependent protein kinase signaling pathways in a late memory consolidation phase of aversively motivated learning in rats. Proc Natl Acad Sci 94: 7041-7046.

Bunzeck N, Dayan P, Dolan RJ, Duzel E. 2010. A common mechanism for adaptive scaling of reward and novelty. Hum Brain Mapp 31: 1380-1394.

Callan DE, Schweighofer N. 2008. Positive and negative modulation of word learning by reward anticipation. Hum Brain Mapp 29: 237-249.

Chun MM, Turk-Browne NB. 2007. Interactions between attention and memory. Curr Opin Neurobiol 17: 177-184.

Duzel E, Yonelinas AP, Mangun GR, Heinze HJ, Tulving E. 1997. Event-related brain potential correlates of two states of conscious awareness in memory. Proc Natl Acad Sci 94: 5973-5978.

Duzel E, Vargha-Khadem F, Heinze HJ, Mishkin M. 2001. Brain activity evidence for recognition without recollection after early hippocampal damage. Proc Natl Acad Sci 98: 8101-8106.
Duzel E, Bunzeck N, Guitart-Masip M, Duzel S. 2010. NOvelty-related motivation of anticipation and exploration by dopamine (NOMAD): Implications for healthy aging. Neurosci Biobehav Rev 34: 660-669.

Fenker DB, Schott BH, Richardson-Klavehn A, Heinze HJ, Duzel E. 2005. Recapitulating emotional context: Activity of amygdala, hippocampus and fusiform cortex during recollection and familiarity. Eur J Neurosci 21: $1993-1999$

Fenker DB, Frey JU, Schuetze H, Heipertz D, Heinze HJ, Duzel E. 2008. Novel scenes improve recollection and recall of words. J Cogn Neurosci 20: $1250-1265$.

Fiorillo CD, Tobler PN, Schultz W. 2003. Discrete coding of reward probability and uncertainty by dopamine neurons. Science 299: 1898-1902.

Frey S, Frey JU. 2008. 'Synaptic tagging' and 'cross-tagging' and related associative reinforcement processes of functional plasticity as the cellular basis for memory formation. Prog Brain Res 169: 117-143.

Frey U, Morris RGM. 1997. Synaptic tagging and long-term potentiation. Nature 385: 533-536.

Frey U, Schroeder H, Matthies H. 1990. Dopaminergic antagonists prevent long-term maintenance of posttetanic LTP in the CA1 region of rat hippocampal slices. Brain Res 522: 69-75.

Frey U, Matthies H, Reymann KG. 1991. The effect of dopaminergic D1 receptor blockade during tetanization on the expression of longterm potentiation in the rat CA1 region in vitro. Neurosci Lett 129: $111-114$.

Goto Y, Grace AA. 2008. Limbic and cortical information processing in the nucleus accumbens. Trends Neurosci 31: 552-558.

Grinband J, Hirsch J, Ferrera VP. 2006. A neural representation of categorization uncertainty in the human brain. Neuron 49: 757-763.

Huang YY, Kandel ER. 1995. D1/D5 receptor agonists induce a protein synthesis-dependent late potentiation in the CA1 region of the hippocampus. Proc Natl Acad Sci 92: 2446-2450.

Krebs RM, Schott BH, Duzel E. 2009. Personality traits are differentially associated with patterns of reward and novelty processing in the human substantia nigra/ventral tegmental area. Biol Psychiatry 65: $103-110$.

Lemon N, Manahan-Vaughan D. 2006. Dopamine D1/D5 receptors gate the acquisition of novel information through hippocampal long-term potentiation and long-term depression. J Neurosci 26: 7723-7729.

Li S, Cullen WK, Anwyl R, Rowan MJ. 2003. Dopamine-dependent facilitation of LTP induction in hippocampal CA1 by exposure to spatial novelty. Nat Neurosci 6: 526-531.

Lisman JE, Grace AA. 2005. The hippocampal-VTA loop: Controlling the entry of information into long-term memory. Neuron 46: 703-713.

Morris RG. 2006. Elements of a neurobiological theory of hippocampal function: the role of synaptic plasticity, synaptic tagging and schemas. Eur J Neurosci 23: 2829-2846.

Niv Y, Daw ND, Joel D, Dayan P. 2007. Tonic dopamine: Opportunity costs and the control of response vigor. Psychopharmacology (Berl) 191: 507-520.

O'Carroll CM, Martin SJ, Sandin J, Frenguelli B, Morris RG. 2006. Dopaminergic modulation of the persistence of one-trial hippocampus-dependent memory. Learn Mem 13: 760-769.

Sajikumar S, Frey JU. 2004. Late-associativity, synaptic tagging, and the role of dopamine during LTP and LTD. Neurobiol Learn Mem 82: 12-25.

Schultz W. 2002. Getting formal with dopamine and reward. Neuron 36: $241-263$.

Schultz W. 2007. Behavioral dopamine signals. Trends Neurosci 30: 203-210.

Tobler PN, Fiorillo CD, Schultz W. 2005. Adaptive coding of reward value by dopamine neurons. Science 307: 1642-1645.

Tulving E. 1985. Memory and consciousness. Can Psychol 26: 1-12.

Vargha-Khadem F, Gadian DG, Watkins KE, Connelly A, Van Paesschen W, Mishkin M. 1997. Differential effects of early hippocampal pathology on episodic and semantic memory. Science 277: 376-380.

Vickery TJ, Jiang YV. 2009. Inferior parietal lobule supports decision making under uncertainty in humans. Cereb Cortex 19: 916-925.

Wittmann BC, Schott BH, Guderian S, Frey JU, Heinze HJ, Duzel E. 2005. Reward-related FMRI activation of dopaminergic midbrain is associated with enhanced hippocampus-dependent long-term memory formation. Neuron 45: 459-467.

Wittmann BC, Schiltz K, Boehler CN, Duzel E. 2008. Mesolimbic interaction of emotional valence and reward improves memory formation. Neuropsychologia 46: 1000-1008.

Yonelinas AP, Otten LJ, Shaw KN, Rugg MD. 2005. Separating the brain regions involved in recollection and familiarity in recognition memory. J Neurosci 25: 3002-3008.

Yu AJ, Dayan P. 2005. Uncertainty, neuromodulation, and attention. Neuron 46: 681-692.

Received August 31, 2010; accepted in revised form March 13, 2011. 


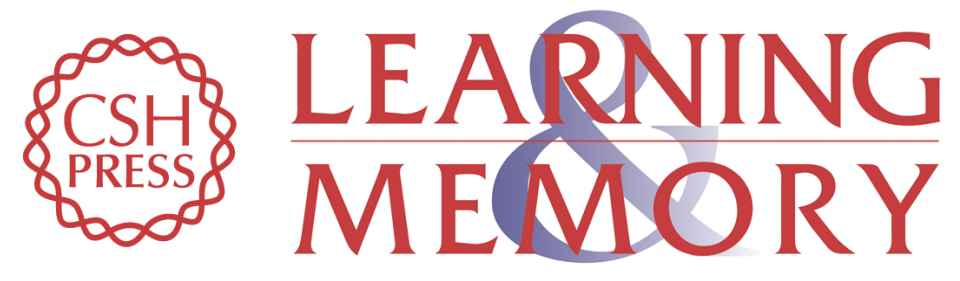

\section{Behavioral specifications of reward-associated long-term memory enhancement in humans}

Bianca C. Wittmann, Raymond J. Dolan and Emrah Düzel

Learn. Mem. 2011, 18:

Access the most recent version at doi:10.1101/lm.1996811

References This article cites 37 articles, 11 of which can be accessed free at: http://learnmem.cshlp.org/content/18/5/296.full.htmI\#ref-list-1

License

Email Alerting Receive free email alerts when new articles cite this article - sign up in the box at the Service top right corner of the article or click here. 